\title{
A Study on Comparison of User's Intention to Use Between Games Operated on Different Platforms (PC and Mobile)
}

\author{
One-hui Choi, Dong-young Shin, Da-som Kim \\ Yonsei University Graduate School of Information, Seoul, Korea
}

\begin{abstract}
Due to a rapid growth of mobile game market, it was found that many successful PC games were conversed into the mobile one and released in the mobile game market. However, there has been a lack of study about comparison among games played in multiple platforms for their intention to use. Thus, in this research, empirical study was done to find out factors that influence the intention to use of the games operated on both PC and mobile platform, and compare and analyze the difference between these platforms. Based on the existing studies related to Technology Acceptance Model (TAM), mobile and PC game, this study proposed four concepts, Service Quality, Social Influence, Instant Connectivity, and System Quality as external variables. In order to verify the model used in this study, it collected 260 survey responses from samples aged between 10 and 40, and statistical analysis was done based on the responses. As a result, it was found that all factors except instant connectivity had an impact on the intention to use in the case of PC platform. In the case of mobile platform, all factors except service quality did influence the intention to use. With this result, it looked into the intention to use of users who play PC and mobile games. In the end, it will suggest what should be focused on for the effective as well as efficient marketing strategy when PC games are conversed into the mobile one.
\end{abstract}

Keywords: multiple platforms (PC and mobile), technology acceptance model expansion, user's intention, games, empirical study, efficient marketing strategy

\section{Growth of Game Market}

Recently, the enterprises progressing toward to jump into the game industry are rapidly increasing due to the various circumstances, such as rapid growth of game industry and market size, developments of various range of game pools, and expansion of game platforms by the advancement and fusion of technology. PC games have risen to its high level of quality by high efficiency of hardware, rapidization of processing speed, development of networks, reaching to the trend of 3D video games and also virtual reality games. The platform is expanded to mobile, mobile games are defined as one of the types of video games accessible to be played in portable devices. It covers the range of all the games which could be reached through portable devices.

One-hui Choi, Ph.D., IT service management, Yonsei University Graduate School of Information, Seoul, Korea.

Dong-young Shin, master, IT service management, Yonsei University Graduate School of Information, Seoul, Korea.

Da-som Kim, master, IT service management, Yonsei University Graduate School of Information, Seoul, Korea.

Correspondence concerning this article should be addressed to One-hui Choi, Yonsei University Graduate School of Information, \#418 New Millennium Hall, 50 Yonsei-ro, Seodaemun-gu, Seoul 120-749, Korea. 


\section{Rapid Growth of Mobile's Game Market}

The recent pattern of consuming propensity of game users shows that the percentage of mobile games is gradually increasing, and due to the continuous improvement of processing speed, games of high performance, also accessible in other platforms are created. Thus, by these characteristics, the mobile games are establishing the new dominant position as new platform. Users are provided with wider range of selecting the games, each possessing its own unique features such as high absorption rate for PC game, and higher accessibility and portability for mobile games. For this reason, the research to examine the main factors affecting the user intention of PC games (online games) and mobile games is regarded to be crucial, but it is hardly accessible to find, yet to be initiated. Thus, this study aims to analyze empirically the factors which have an influence of user intention on PC games and mobile games and suggest the direction of what aspects the developments and customer service should be focused on two areas, PC and mobile games. Based on applying the TAM (Technology Acceptance Model) on PC and mobile games, this study established the research model and empirically analyzed it. For the model, referring to the TAM suggested by Davis (1989) and existing research literature, the study extracted factors that affect the user selection on games. Also, considering the mutuality of extracted factors, the study suggested the model of PC and mobile game uses and analyzed the results.

\section{Literature Review}

\section{Mobile Games}

Mobile games can be defined as the types of games played in portable devices, such as cell phone or PDA, logging into the servers through wireless internet (Kim, Han, \& Seo, 2005). The early stages of mobile games were confined to the certain traditionally classical games and simple puzzle types rather than brand-new or creative games, due to the limited platforms. However, by the spread of smartphones and improvement of efficiency of devices, mobile games began to introduce higher level of quality subjects, surpassing the level of PC game versions (Gwon, Kim, \& Choi, 2011).

Mobile games possess higher accessibility and instant connectivity, due to the large pool of users using mobile devices especially smartphones and comparing to the former traditional game platforms, it is largely differentiated in the characteristic of portability, convenience. Factors such as accessibility and convenience act as replay factors to attract more users to the user pool, affecting the expansion of market size. More users can enjoy in a short period of their flexible time when moving, thus resulting into thick client base.

\section{Technology Acceptance Model (TAM)}

Information technology acceptance model is the structure based on TRA (theory of reasoned action) on social psychology to analyze and predict the behaviors of information technology users (Davis, 1989). This model is the expansion of relationship of certain behavioral attitude to certain action and intention of action suggested on TRA, for the study of information technology selection of information technology users (Adams, Nelson, \& Todd, 1992; Davis, Bagozzi, \& Warshaw, 1989). The representative theory, TAM was proposed by Davis (1989), and it is recognized to be a highly persuasive and simple model for explaining the information technology acceptance and behaviors of users. Also, it provides the theoretical basis for the further expanded research. Especially, TAM is regarded to be highly explanatory and reliable through numerous empirical researches, on the subject of acceptance of advanced technology by the users. Davis (1989) suggested perceived usefulness and perceived ease of use for the crucial factors of using information system for users. 
Perceived usefulness means the degree to which a person believes that particular information system would enhance his job performance, and perceived ease of use is defined as the degree to which a person believes that using a particular system would alleviate the effort needed. Davis (1989) asserted that these two factors would be influential on measuring the acceptance of information system. In other words, perceived usefulness and perceived ease of use affect the attitude towards use and intention to use, and intention to use influence the actual system use.

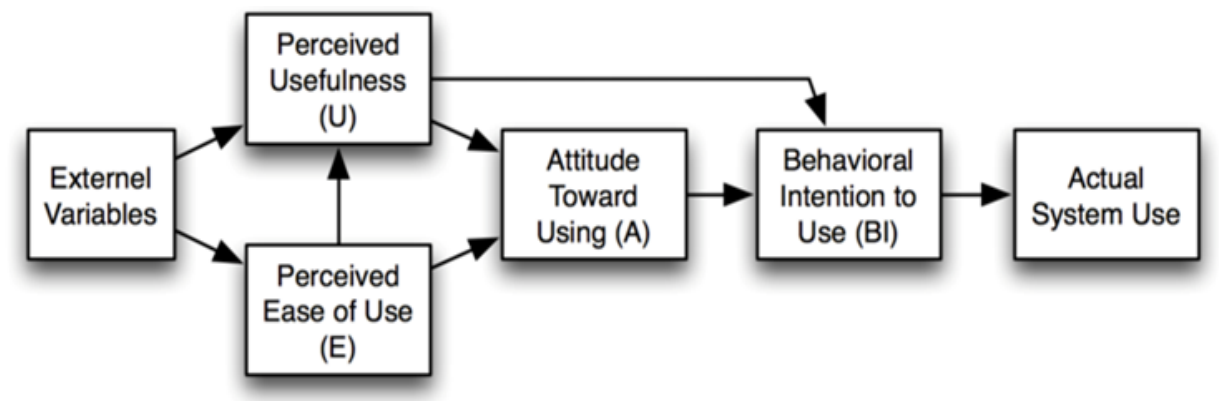

Figure 1. Technology acceptance model.

\section{Factors Influencing the Use of Products and Services}

Ajzen (1991), in his Theory of Planned Behavior (TPB), asserted that the factors such as attitudes, norms, or behavioral controls affect one's intention of using products and services, and this intention acts as a catalyst for the user to actually utilize in their behavior. The model Ajzen (1991) suggested is widely applied to information system field, also in marketing area.

DeLone and McLean (1992) proposed that a number of factors including the quality of the system and processed information have a direct impact on the general system usability and degree of satisfaction of the users. Accordingly, users, in regards to the use of information system, are largely affected to the elements, such as usability, availability, usefulness, quality of system, and social impact.

\section{Research Model and Hypotheses}

\section{Research Model}

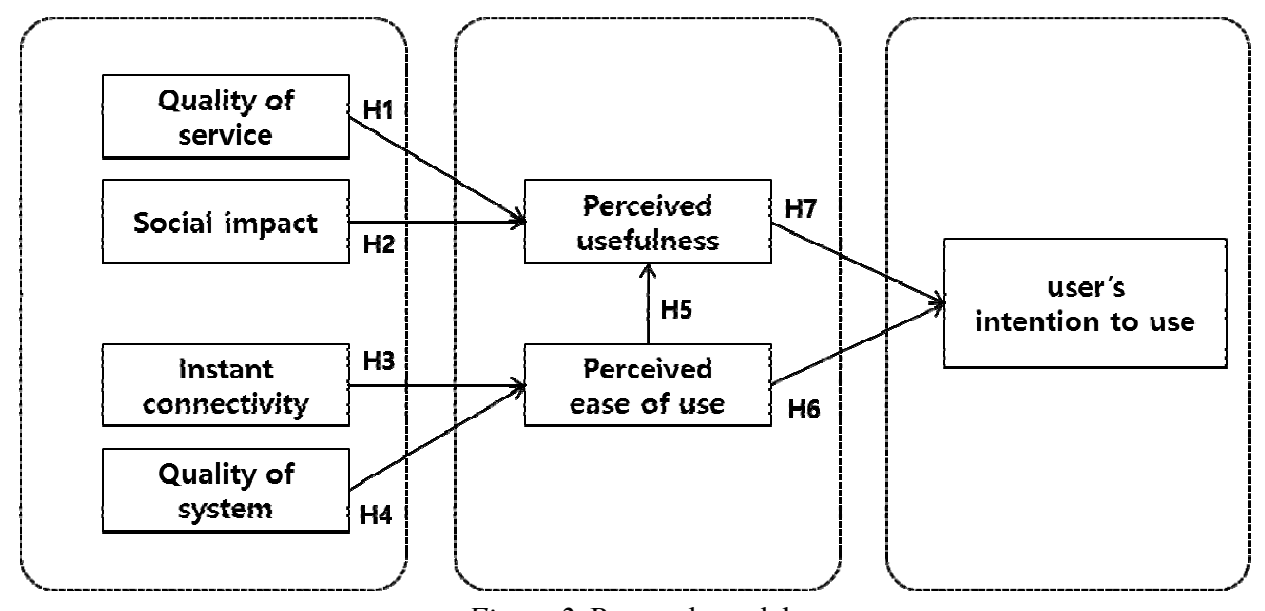

Figure 2. Research model.

This study established a research model to derive the utilization intention of platform-specific users, applying to multi-platform (PC, mobile) games, based on the structural framework of TAM. Independent 
variables are classified as system-related, personal, and social factors, on the basis of studies related to TAM expansion. For each classified area, among the factors that are validated in existing literature, this study extracted the specific types that can compare the common characteristics of PC and mobile games and also, the intention of users based on different platforms. Thus, quality of service, quality of system, and instant connectivity on system-related factor, innovativeness on social factor, and social impact on social factor were selected. Research model of this study is as follows.

\section{Quality of Service}

Venkatesh and Davis (2000) have defined the degree of perceiving how well one can perform their task by utilizing service in TAM2 as the level of quality of the output. This study substituted the quality of perceived output as quality of service, taking this as external variable which affects the perceived usefulness and the assumed quality of service would exert influence on the service usefulness and level of utility.

H1. The quality of service will have a positive effect on the perceived usefulness.

\section{Social Impact}

Rogers (1995) initiated an experiment which compares the early adopters with late adopters, in the process of adapting the new technologies. This experiment concluded that for early adapters, factors such as mass media influenced on the level of acceptance of new technologies, and for late adapters, they are more inclined to be influenced by the acquaintances. As the study suggests, the social impact acts as an crucial factor such as in the selection and purchase of external goods and services, and reception of information system. Therefore, this study set up the following hypothesis.

H2. Social impact will have a positive effect on perceived usefulness.

\section{Instant Connectivity}

Instant connectivity is the characteristic of mobile games, instantly connected to the game easily and quickly regardless of time and place via mobile (terminal) (Müller-Veerse, 2000). For the external factor which could strengthen the comparative study of platform-specific user's intention to use, assuring that it would be appropriate to add the characteristics of mobile, instant connectivity is added and hypothesized.

H3. Instant connectivity will have a positive effect on perceived ease of use.

\section{Quality of System}

Quality of system is the degree to which a user can use a reliable and efficient system (Dey, 2001). As the technologies have developed through, quality of system acts as an essential cause on the selection process of users. According to the study on success of mobile games, the speed and reliability of the system were proposed to be the influential factors (Dodds \& Monroe, 1985).

H4. Quality of system will have a positive effect on the perceived ease of use.

\section{Perceived Usefulness, Perceived Ease of Use, User's Intention to Use}

The perceived usefulness and the perceived ease of use are important concepts configuring TAM. Davis, Bagozzi, and Warshaw (1989) suggested these two factors as the major parameters of reception behaviors of computer. The perceived usefulness means "the subjective expectations about the degree of enhancement of work performance by utilizing the information technology". Perceived ease of use is defined to be "the expectations on the degree of not requiring one's effort for utilization of information technology". In other words, the ease of use focuses on the instrumental aspect, enhancing the work performance by applying the 
technology in high level of ease of use than the lower level. Thus, TAM suggested that perceived ease of use affects perceived usefulness, but the reverse is not established. Davis (1989) concluded that the perceived usefulness has a significant impact on user's intention to use.

H5. Perceive ease of use will have a positive effect on perceived usefulness.

H6. Perceived ease of use will have a positive effect on the user's intention to use.

H7. Perceived usefulness will have a positive effect on user's intention to use.

\section{Research Methods}

\section{Sample Design}

This study set up the questionnaire survey target at the age of 10-40 whose use of multi-platform games is high. The survey was conducted for approximately one month, kicking-off in the beginning of November. The 260 samples of collected data by convenience sampling were utilized for data analysis due to the limitation of time and cost. The percentage of gender from valid samples is categorized as male $65.8 \%$, female $34.2 \%$, more respondents from male and the age percentage were distributed as $10 \mathrm{~s} 37.7 \%, 20 \mathrm{~s} 37.7 \%$, 30s $21.5 \%$, and $40 \mathrm{~s}$ $3.1 \%$.

\section{Metric Design}

This study installed the model of Likert-type seven point scale for metrics of each concept. The metrics were modified considering on the context of comparative studies of intention of each platform-specific users based on the existing literature.

\section{Results of the Research}

This study applied Smart PLS2 program for statistical data processing. The internal consistency analysis of list of concepts was measured by Cronbach's $\alpha$ coefficient obtained through reliability analysis. The validity of measure tools was verified by confirmatory factor analysis. In order to verify the hypothesis, the regression analysis was conducted.

\section{Reliability Analysis}

The reliability of each concept was verified by the Cronbach's $\alpha$ coefficient for internal consistency. The results of reliability analysis of comparative study on measuring the intention of PC-platform user conclude: quality of service 0.813 , instant connectivity 0.706 , quality of system 0.736 , perceived usefulness 0.622 , perceived ease of use 0.856 , user's intention to use 0.800 , measured to be higher than 0.6 which is the general acceptance level. Also, the results of reliability analysis of comparative study of measuring the intention of mobile-platform user conclude: quality of service 0.816 , instant connectivity 0.755 , quality of system 0.761 , perceived usefulness 0.492 , perceived ease of use 0.810 , and user's intention to use 0.746 , also measured to be higher than 0.6 which is the general acceptance level. However, the social impact of both the PC and mobile platform were measured as $0.465,0.39$, and perceived usefulness of mobile platform as 0.492 , these confidence coefficients scoring lower than the general acceptance level.

\section{Feasibility Analysis}

This study performed exploratory feasibility analysis by main factor analysis using Varimax rotation, utilizing IBM SPSS Statistics 21. The factor was extracted based on the Eigenvalue and unrotated factor solution. Establishing the criteria for valid factor as having the Eigenvalue of 1.0 or more, the five factors were 
extracted in the PC platform, reaching overall explanatory power of $61.45 \%$. Further, mobile platform was proposed to extract six factors, reaching the overall explanatory power $63.23 \%$. The factors less than 0.5 loads have been skipped. The results of exploratory feasibility analysis show the limitations of perceived ease of use, which is the major factor of TAM, providing insufficient description on one of the variables. Thus, the study conducted confirmatory factor analysis through Smart PLS.

\section{Comparative Study Using PC Platform}

The results of regression analysis for the verification of hypotheses are provided in Figure 3. For the regression analysis, factor scores for each concept were figured and utilized for regression analysis, under the significance level 95\%, scored of $P$-value 0.05 .

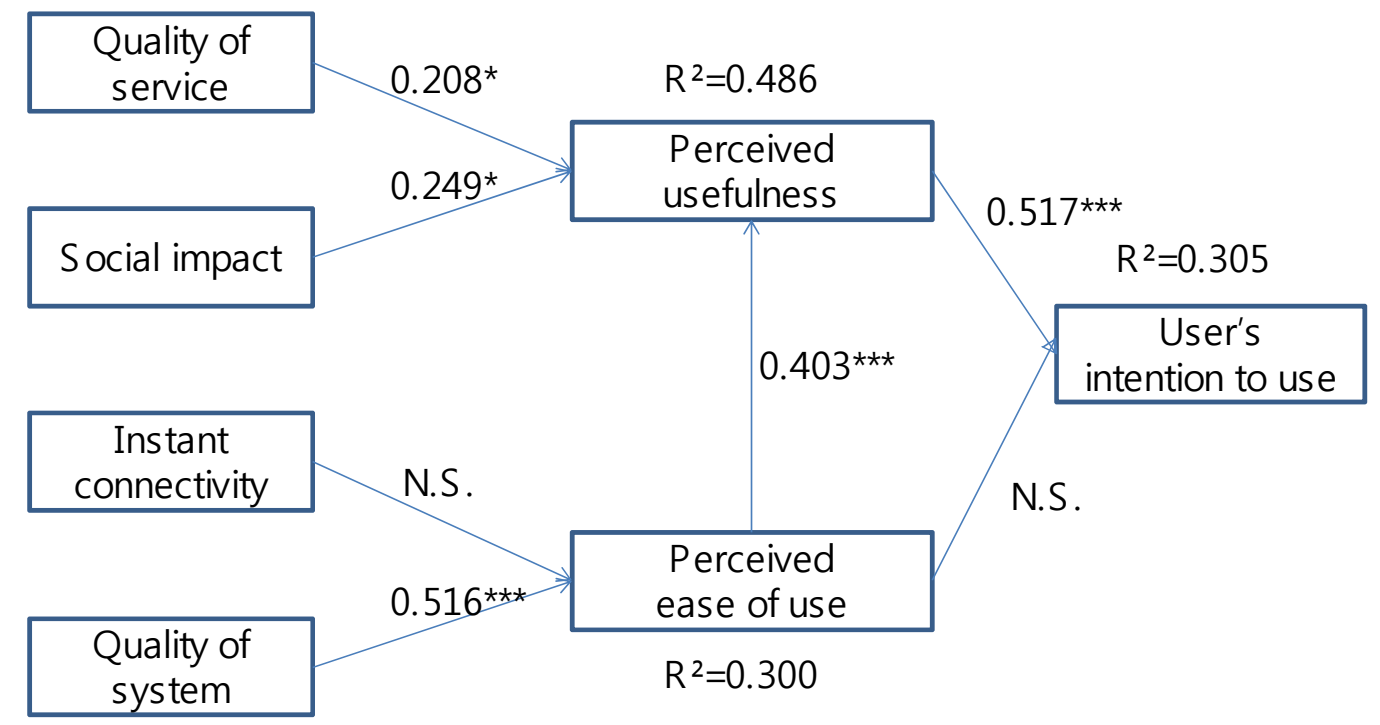

Figure 3. PC platform.

H1. The quality of service will have a positive effect on the perceived usefulness. (Supported)

H2. Social impact will have a positive effect on perceived usefulness. (Supported)

H3. Instant connectivity will have a positive effect on perceived ease of use. (Rejected)

H4. Quality of system will have a positive effect on the perceived ease of use. (Supported)

H5. Perceive ease of use will have a positive effect on perceived usefulness. (Supported)

H6. Perceived ease of use will have a positive effect on the user's intention to use. (Rejected)

H7. Perceived usefulness will have a positive effect on user's intention to use. (Supported)

\section{Comparative Study Using Mobile Platform}

The results of regression analysis for verifying the hypotheses are followed as Figure 4. For the regression analysis, factor scores for each concept were figured and utilized for regression analysis, under the significance level 95\%, scored of $P$-value 0.05 .

H1. The quality of service will have a positive effect on the perceived usefulness. (Rejected)

H2. Social impact will have a positive effect on perceived usefulness. (Supported)

H3. Instant connectivity will have a positive effect on perceived ease of use. (Supported)

H4. Quality of system will have a positive effect on the perceived ease of use. (Supported)

H5. Perceive ease of use will have a positive effect on perceived usefulness. (Supported) 
H6. Perceived ease of use will have a positive effect on the user's intention to use. (Rejected)

H7. Perceived usefulness will have a positive effect on user's intention to use. (Supported)

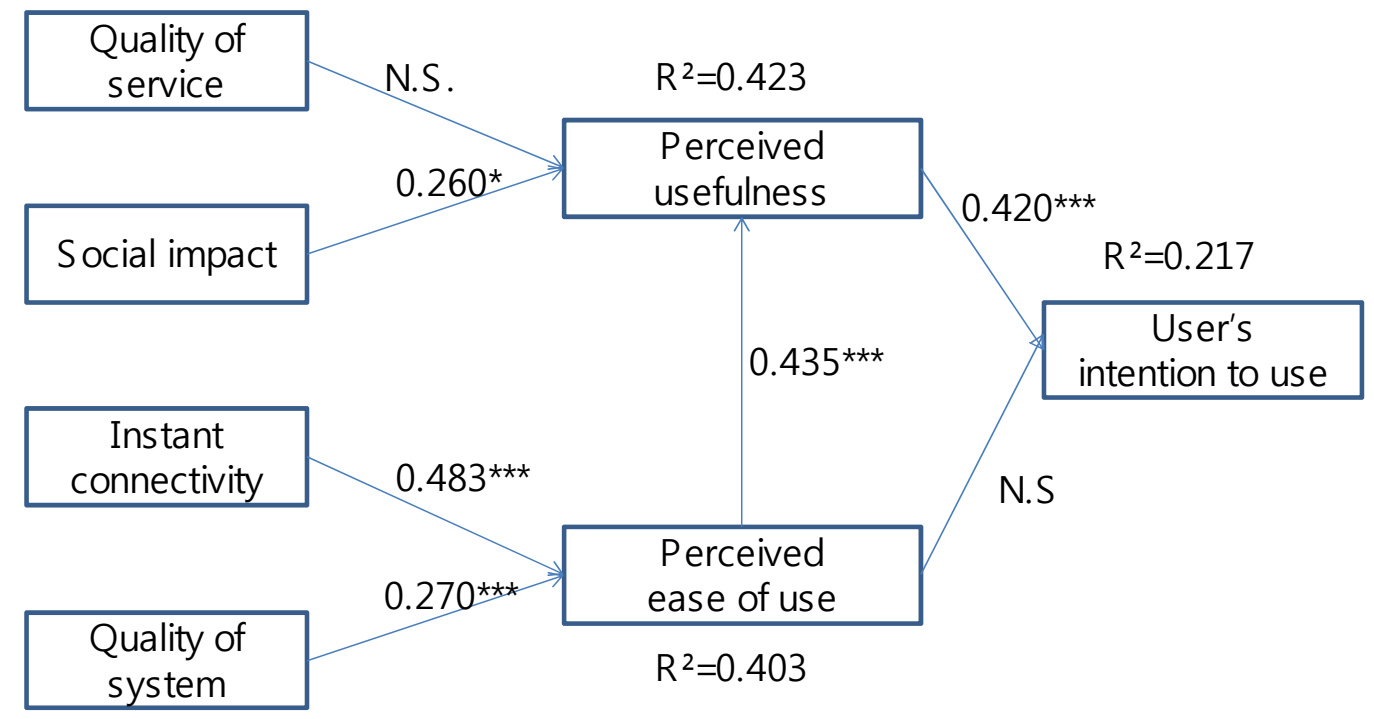

Figure 4. Mobile platform.

\section{Discussion and Conclusion}

\section{Summary and Implications of the Research}

This study, based on the existing researches on TAM, PC games, and mobile games, examined the factors that affect the user's intention to use of multi-platform games, both on PC and mobile and checked whether the difference of factors exists. Considering the number of examples showing the trend of successful PC games trying for the conversion into mobile games market, it was hardly found to comparatively study the user's intention to use of multi-platform games. This study can provide the initial and proper implication considering the situation.

Based on the conclusions of this study, the implications are as follows. First, quality of service was verified to be the affective factor for perceived usefulness, and there is no much variability between PC and mobile platforms. Thus, providing the high quality and reliable game service both on PC and mobile platform is the utmost point and immediate response is required if a disturbance arises. In the PC platform, quality of system was proved to be a valid factor for the effect of perceived ease of use and for the mobile platform, quality of system, and instant connectivity were the valid factors.

Concerning these results, if a company is planned to initiate the conversion process from PC games to mobile games, the subject needs to be simple, possible to enjoy for a short period of time, less dissatisfactory even if the effect of sound and graphics is lessened, rather than difficult progress or content, protracted duration, and fancy sound and graphics. In reverse, if mobile games are targeted to be converged to PC platform, there needs to be developments on the aspect of sound and graphics.

To interpret in terms of why the perceived ease of use does not have a direct impact on user's intention to use, it can be assumed that user's intention to use could not be raised due to the sole reason of easy operational aspect or content, considering the nature of wide range of game choices. This could be backed up by the point that complex structure-based games in the past and present gained high number of user pool. 
To summarize the implications of this study above, this study academically revealed the applicability of TAM model on the field, initiating the comparative study of influential factors of user's intention to use on multi-platform games. There is a contributing point of this study, confirming that the direct impact of perceived ease of use to user's intention to use can be denied. Also practically, in the process of conversing PC games to mobile games and vice versa, implications on what kinds of game should be targeted, and what aspects the game should possess and focus to be reconfigured were provided for the business fields.

\section{Limitations of the Study and Suggestions for the Future Research}

The directions for the future research are as follows, based on the limitations explored of this research. First, the lack of statistical techniques could not draw out the differences between the factors that affect the user's intention to use on each game platform, PC and mobile. The future research needs to be analyzed through sophisticated statistical techniques on comparing the direct difference on the factors that affect the user's intention to use on two models. Second, the study presented limited scope of four external variables which affect the perceived usefulness and perceived ease of use. Also, social impact, which is one of the variables failed to pass the reliability test and the verification of the hypothesis could not be carried out. There is a need for further additional effort on measuring the social impact and for further research by discovering new factors additionally, thus broadening the research area of user's intention to use of multi-platform games.

\section{References}

Adams, D. A., Nelson, R. R., \& Todd, P. A. (1992). Perceived usefulness, ease of use, and usage of information technology: A replication. MIS Quarterly, 16(2), 227-247.

Davis, F. D. (1989). Perceived usefulness, perceived ease of use, and user acceptance of information technology. MIS Quarterly, 13(3), 319-340.

Davis, F. D., Bagozzi, R. P., \& Warshaw, P. R. (1989). User acceptance of computer technology: A comparison of two theoretical models. Management Science, 35(8), 982-1002.

Dey, A. K. (2001). Understanding and using context. Personal and Ubiquitous Computing, 5, 4-7.

Dodds, W. B., \& Monroe, K. B. (1985). The effect of brand and price information on subjective product evaluations. Advances in Consumer Research, 12, 85-90.

Müller-Veerse, F. (2000). Mobile commerce report. Retrieved from http://www.durlacher.com/downloads/mcomreport.pdf

Gwon, H., Kim, H., \& Choi, Y. (2011). An emprical study applying structural equation on the continued intention to use and recommendations of the smartphone games. Journal of Korea Game Society, 11, 115-125.

Kim, H., Han, C., \& Seo, B. (2005). A Study on factors affecting the intention to use of mobile games. Korea e-Business Studies, 10, 1-19.

Ajzen, I. (1991). The theory of planned behavior. Organizational Behavior and Human Decision Processes, 50, $179-211$.

Rogers, E. M. (1995). Diffusion of innovations (4th ed.). New York: Free Press.

Venkatesh, V., \& Davis, F. D. (2000). A theoretical extension of the technology acceptance model: Four longitudinal field studies. Management Science, 46, 186-204.

Delone, W. H., \& Mclean, E. R. (1992). Information system success: The quest for the dependent variable. Information System Research, 3, 60-95. 